\title{
Acute Rheumatic Pericarditis
}

National Cancer Institute

\section{Source}

National Cancer Institute. Acute Rheumatic Pericarditis. NCI Thesaurus. Code C35200.

Inflammation of the pericardium in acute rheumatic heart disease. 